\title{
gु \\ Optical initialization, readout, and dynamics of a Mn spin in a quantum dot
}

\author{
C. Le Gall, ${ }^{1}$ R. S. Kolodka, ${ }^{1}$ C. L. Cao, ${ }^{1,2}$ H. Boukari, ${ }^{1}$ H. Mariette,,${ }^{1}$ J. Fernández-Rossier, ${ }^{2}$ and L. Besombes ${ }^{1, *}$ \\ ${ }^{1}$ CEA-CNRS Group "Nanophysique et Semiconducteurs," Institut Néel, CNRS-Université Joseph Fourier, \\ 25 Avenue des Martyrs, 38042 Grenoble, France \\ ${ }^{2}$ Departamento de Física Aplicada, Universidad de Alicante, San Vicente del Raspeig 03690, Spain
}

(Received 12 March 2010; revised manuscript received 17 May 2010; published 17 June 2010)

\begin{abstract}
We have investigated the spin preparation efficiency by optical pumping of individual Mn atoms embedded in CdTe/ZnTe quantum dots. Monitoring the time dependence of the intensity of the fluorescence during the resonant optical pumping process in individual quantum dots allows to directly probe the dynamics of the initialization of the Mn spin. This technique presents the convenience of including preparation and readout of the Mn spin in the same step. Our measurements demonstrate that Mn spin initialization, at zero magnetic field, can reach an efficiency of $75 \%$ and occurs in the tens of nanoseconds range when a laser resonantly drives at saturation one of the quantum-dot transition. We observe that the efficiency of optical pumping changes from dot-to-dot and is affected by a magnetic field of a few tens of millitesla applied in Voigt or Faraday configuration. This is attributed to the local strain distribution at the Mn location which predominantly determines the dynamics of the Mn spin under weak magnetic field. The spectral distribution of the spin-flip-scattered photons from quantum dots presenting a weak optical pumping efficiency reveals a significant spin relaxation for the exciton split in the exchange field of the Mn spin.
\end{abstract}

DOI: 10.1103/PhysRevB.81.245315

PACS number(s): 78.67.Hc, 78.55.Et

\section{INTRODUCTION}

The ability to control spins in semiconductors nanostructures is an important issue for spintronics and quantuminformation processing. Single spin detection and control is a key but very challenging step for any spin-based solid-state quantum-computing device. In the last few years, efficient optical techniques have been developed to control the spin of individual carriers ${ }^{1}$ or ensemble of nuclei ${ }^{2}$ in semiconductor quantum dots (QDs). Thanks to their expected long spin coherence time, magnetic atoms in a semiconductor host could be an alternative media to store quantum information in the solid state. However, as these localized spins interact weakly with their environment, they can be hardly controlled by electrical or optical methods. Recently QDs containing individual $\mathrm{Mn}$ atoms have been realized both in II-VI (Ref. 3) and III-V (Ref. 4) compounds. In these systems, since the confined carriers and Mn spin functions become strongly mixed, the resonant optical excitation of the QD strongly affects the spin state of $\mathrm{Mn}$ atom offering a possibility of full optical control. ${ }^{5}$

When a Mn atom is included in a II-VI semiconductor QD (CdTe in $\mathrm{ZnTe}$ ), the spin of the optically created electron-hole pair (exciton) interacts with the five $d$ electrons of the Mn (total spin $S=5 / 2$ ). This leads to a splitting of the once simple photoluminescence (PL) spectrum of an individual QD into six $(2 S+1)$ components. This splitting results from the spin structure of the confined heavy holes which are quantized along the QDs' growth axis with their spin component taking only the values $J_{z}= \pm 3 / 2$. In first approximation, the hole-Mn exchange interaction reduces to an Ising term $J_{z} \cdot S_{z}$ and shifts the emission energy of the QD, depending on the relative projection of the Mn and hole spins. ${ }^{6}$ As the spin state of the Mn atom fluctuates during the optical measurements, the six lines are observed simultaneously in time average PL spectra. The intensities of the lines reflect the probability for the Mn to be in one of its six spin components and the PL is a probe of the spin state of the Mn when the exciton recombines. ${ }^{7}$

In this paper, we show that one can exploit the absorption of an individual II-VI QD to optically initialize the spin state of an embedded $\mathrm{Mn}$ atom. We use resonant optical excitation of one of the six exciton levels of a Mn-doped QD to prepare by optical pumping the spin state of the magnetic atom. Under these resonant excitation conditions, scattered photons coming from spin flips of the exciton without change in the $\mathrm{Mn}$ spin are observed. This fluorescence signal is used to probe the dynamics of the initialization of the Mn spin during the optical pumping process. A pumping efficiency of about $75 \%$ is obtained with an initialization time in the tens of nanoseconds range. A saturation of the optical pumping efficiency is observed at high excitation power reflecting the nonlinear absorption of the QD exciton state. We observed that the efficiency of optical pumping is affected by a magnetic field of a few tens of millitesla applied in Voigt or Faraday configuration and that it can be very weak at zero field for some of the QDs. This reveals the influence of the $\mathrm{Mn}$ fine structure on the spin dynamics: the magnetic anisotropy of the Mn spin induced by biaxial strains slows down the relaxation at zero magnetic field. Partial relaxation of the strain or an anisotropic in-plane strain distribution at the $\mathrm{Mn}$ spin location restores coherent precession of the Mn spin away from the optical axis decreasing the efficiency of the optical pumping signal. The spectral distribution of the photons scattered by the QD shows that an efficient exciton spin relaxation occurs within the exciton-Mn (X-Mn) complex. This spin relaxation is attributed to the large single-phonon mediated spin flip for an exciton split in the exchange field of the Mn.

The manuscript is divided in six sections: in Sec. II we give details about the Mn-doped QD samples and the timeresolved optical pumping experiments. In Sec. III we de- 
scribe how angular momentum is transferred from circularly polarized light to a Mn spin in a QD. In Secs. IV and V we show how the time resolved and $\mathrm{cw}$ resonant fluorescence signal can be used to probe the dynamics of the exciton and Mn spins. In Sec. VI we present a simple model for the optical pumping process and discuss the mechanism controlling the Mn spin dynamics.

\section{SAMPLES AND EXPERIMENT}

The samples used in this study are all grown on $\mathrm{ZnTe}$ substrates and contain $\mathrm{CdTe} / \mathrm{Zn}_{0.8} \mathrm{Mg}_{0.2} \mathrm{Te}$ QDs. A 7.5monolayer-thick CdTe layer is deposited at $280{ }^{\circ} \mathrm{C}$ by atomic layer epitaxy on a $\mathrm{Zn}_{0.8} \mathrm{Mg}_{0.2} \mathrm{Te}$ barrier grown by molecular beam epitaxy at $360{ }^{\circ} \mathrm{C}$. The dots are formed by the well-established Tellurium deposition/desorption ${ }^{8}$ process and protected by a $100-\mathrm{nm}$-thick $\mathrm{Zn}_{0.8} \mathrm{Mg}_{0.2}$ Te top barrier. The incorporation of $\mathrm{Mg}$ in the barriers leads to a stronger confinement of the holes and consequently to an increase in their exchange interaction with the Mn spin. The height of the QDs' core is of few nanometers and their diameter is in the $10-20 \mathrm{~nm}$ range. $\mathrm{Mn}$ atoms are introduced during the growth of the CdTe layer. The Mn concentration is adjusted to optimize the probability to detect one Mn per dot. ${ }^{9}$

During this growth process, the position of the magnetic atom, the shape of the QD and the strain at the Mn location are not controlled. In most of the dots, the presence of an anisotropic strain distribution in the QD plane produces a mixing of light-hole (lh) and heavy-hole (hh) subbands, as described with the Bir-Pikus Hamiltonian. This valence-band mixing $(\mathrm{VBM})$ is responsible for a linear polarization degree of the QD emission with a linear polarization direction imposed by the strain distribution. ${ }^{10}$ In QDs containing a magnetic atom, the VBM affects the coupling between the confined hole and the localized magnetic moments. It allows simultaneous hole-Mn spin flips responsible for a coupling between the dark and bright exciton states at zero magnetic field. This is responsible for the appearance of additional PL lines (more than six) on the low-energy side of the emission spectrum of most of the Mn-doped QDs (Ref. 6) as will be detailed in Sec. V.

Optical addressing of QDs containing a single magnetic atom are achieved using microspectroscopy techniques. High refractive index hemispherical solid immersion lens are mounted on the bare surface of the sample to enhance the spatial resolution and the collection efficiency of single-dot emission in a low-temperature $(T=5 \mathrm{~K})$ scanning optical microscope. ${ }^{11}$ This technique reduces the reflected and scattered light at the sample surface and decrease the focal spot area allowing the measurement of spin-flip-scattered photons from an individual Mn-doped QD. ${ }^{12,13}$ Despite the quite large QD density and the large number of dots in the focal spot area, single QD transitions can be identified by their spectral signatures (see inset of Fig. 2). A weak magnetic field (a few tens of millitesla) can be applied in Voigt or Faraday configuration using either permanent magnets or superconductive coils.

In the time-resolved optical pumping experiments presented in this paper, single QD PL is quasiresonantly (probe) and resonantly (pump) excited with two tunable cw dye lasers. Trains of resonant light pulses with variable duration and wavelength are generated from the cw lasers using acousto-optical modulators with a switching time of about 10 ns. The circularly polarized collected PL is dispersed by a 1-m double monochromator before being detected by a fast avalanche photodiode in conjunction with a time-correlated photon-counting unit with an overall time resolution of about 50 ps.

\section{RESONANT OPTICAL PUMPING OF A SINGLE MN SPIN}

Recently, the optical orientation of a single magnetic atom by the injection of spin-polarized carriers in a Mn-doped II-VI QD has been demonstrated. ${ }^{14}$ In this experiment, spinpolarized excitons were generated on a QD excited state and, after relaxation, a nonequilibrium $\mathrm{Mn}$ spin population was produced by a thermalization among the levels of the exciton-Mn ground states. However, as proposed by Govorov et al., ${ }^{15}$ the direct resonant excitation of one optical transition of the ground X-Mn complex could be used to perform a direct and more efficient optical pumping of the Mn spin. In this optical pumping process, a laser drives resonantly one of the exciton-Mn transition $\left(\left|-1, S_{z}=-5 / 2\right\rangle\right.$ in Fig. 1) with a Rabi frequency $\Omega=\mathcal{P E} / \hbar$ with $\mathcal{P}$ the dipolar moment of the QD transition and $\mathcal{E}$ the amplitude of the electric field of the resonant laser. A photon absorption occurs only if the Mn spin in the QD is in the $S_{z}=-5 / 2$ spin state. The resultant exciton can radiatively recombine via the same channel or a relaxation process can project the X-Mn complex in a state with $S_{z} \neq-5 / 2$. After a few cycles of absorption emission, the probability of detecting the $\mathrm{Mn}$ in the $S_{z}=-5 / 2$ state decreases. In this mechanism, we have assumed that the Mn spin was conserved once the exciton has recombined. The conservation of the Mn spin between the recombination of an exciton and the absorption of a photon can be altered in two ways: either by a relaxation process involving an exchange of energy or by a coherent evolution. ${ }^{14,16}$ A coherent evolution can be neglected if the fine structure of the $\mathrm{Mn}$ atom is dominated by a magnetic anisotropy along the growth axis. ${ }^{14}$ Otherwise, processes such as the coherent evolution of the Mn spin in the hyperfine field of the Mn nucleus or in the tetragonal crystal field leads to a change in the Mn spin state between the injection of two excitons. In that case, no optical pumping can occur. In the fallowing, we will use $\Gamma_{\mathrm{Mn}, G}$ to describe the characteristic rate at which the Mn spin state changes due to coherent or incoherent processes, when the QD is empty. $\Gamma_{\mathrm{Mn}, \mathrm{X}}$ will correspond to the relaxation rate of the $\mathrm{Mn}$ interacting with an exciton. This mechanism of Mn spin manipulation is similar to the pumping process used to prepare a single carrier spin in a QD. ${ }^{17,18}$ It involves a forbidden transition (i.e., spin flip of the Mn interacting with the exciton) and is based on the inequality $\Gamma_{\mathrm{Mn}, \mathrm{X}}>\Gamma_{\mathrm{Mn}, G}$.

To demonstrate and test the efficiency of this optical pumping process, we developed a two wavelength pumpprobe setup allowing an optical initialization and readout of the Mn spin. In this experiment, a resonant circularly polar- 


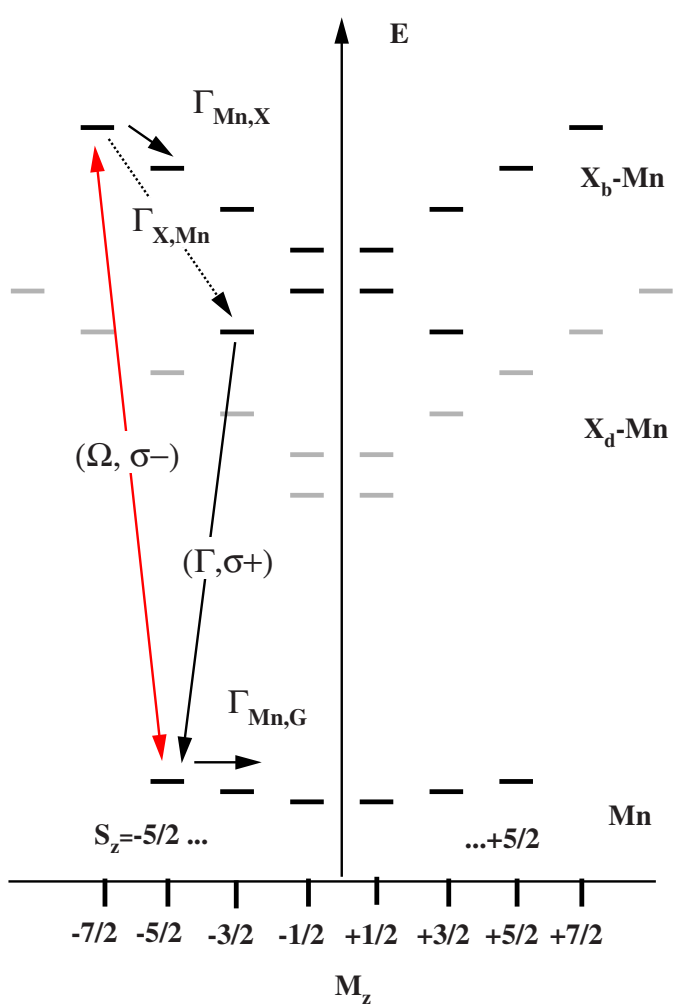

FIG. 1. (Color online) Energy levels of a Mn-doped QD involved in the optical pumping mechanism described in the text [black: bright excitons $\left(X_{b}\right)$; gray: dark excitons $\left(X_{d}\right)$ ]. The sates are displayed as a function of their total angular momentum $M_{z}$ and energy $E$. For the resonant optical pumping, the QD is resonantly driven on the state $S_{z}=-5 / 2$ with a $\sigma$ - laser pulse with a Rabi frequency $\Omega$. The scattered photons obtained after a spin flip of the exciton (rate $\Gamma_{\mathrm{X}, \mathrm{Mn}}$ ) are recorded in $\sigma+$ polarization. The intensity of the $\sigma+\mathrm{PL}$ detected on the low-energy bright exciton level is proportional to the population of $S_{z}=-5 / 2$ and is used to probe the optical pumping efficiency.

ized cw laser (resonant pump) tuned on an X-Mn level pumps the Mn spin. In the initial state at $t=0$, the Mn atom is in thermal equilibrium (i.e., all the six spin states are equally populated). The resonant creation of an exciton followed by a spin relaxation of the $\mathrm{Mn}$ in the exchange field of the exciton empties the spin state under excitation: the probability of occupation of this spin state decreases over time. Then, a second laser train, linearly polarized and tuned on an excited state of the QD (quasiresonant probe), injects excitons independently of the Mn spin state $S_{z}$. Spin relaxation of the $\mathrm{X}-\mathrm{Mn}$ complex under these conditions of excitation drives the $\mathrm{Mn}$ atom back to an equilibrium where all spin states are equally populated. Recording one of the six PL lines under this periodic sequence of excitation, we monitor the time evolution of the probability of occupation of a given Mn spin state.

The main features of this experiment are presented in Fig. 2 . In this example, $\sigma+\mathrm{PL}$ signal is recorded on the lowenergy X-Mn line. The QD is resonantly excited on the highenergy state of the X-Mn complex with $\sigma$ - photons. This excitation can only create an exciton in the dot if the Mn spin state is $S_{z}=-5 / 2$. This selective absorption combined with a

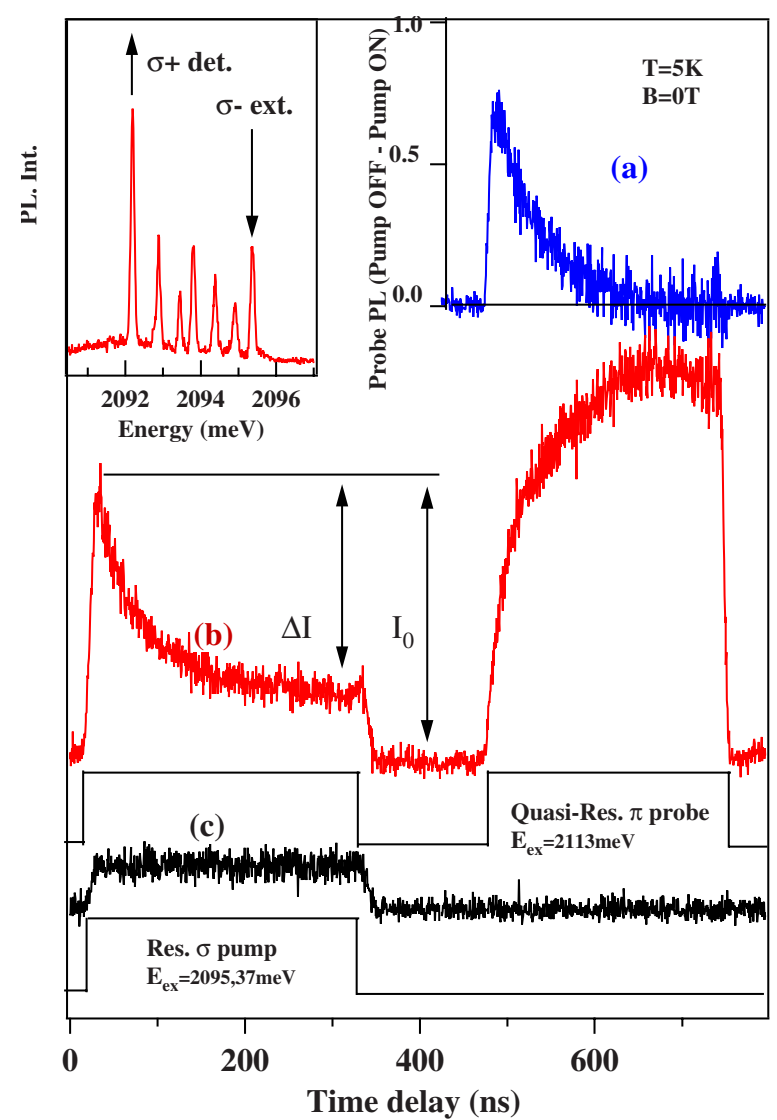

FIG. 2. (Color online) PL transients recorded on the low-energy line of a Mn-doped QD (QD1) under the quasiresonant (QD excited state: probe) and resonant (QD ground state: pump) optical excitation sequence displayed at the bottom. The inset presents the QD PL under nonresonant excitation and the configuration of the resonant excitation and detection. (a) Difference between the PL produced by the probe when the pump is OFF and when the pump is $\mathrm{ON}$, (b) PL from the pump and the probe, and (c) resonant PL produced by the pump alone. Because of the Mn spin memory in the absence of injected carriers, no signature of pumping is observed when the linearly nonresonant probe is OFF (c). The optical pumping process is directly observed on the resonant fluorescence produced by the pump and latter on the PL from the probe laser. $I_{0}$ is the amplitude of the fluorescence at the beginning of the pump pulse and $\Delta I$ the amplitude of the transient. $\Delta I / I_{0}$ is the efficiency of the spin optical pumping.

spin relaxation for the X-Mn complex are responsible for the optical pumping process that progressively decreases the population of the state $S_{z}=-5 / 2$. After this pumping sequence, the resonant pump laser is switched off and followed by a linearly polarized excitation on an excited state (quasiresonant probe). The amplitude of this quasiresonant PL depends on the population of $S_{z}=-5 / 2$ and, at the beginning of the probe pulse, is a probe of the resonant pumping efficiency reached at the end of the pump pulse.

This is illustrated in Fig. 2(a) which presents the difference of the two PL signals produced by the probe when the resonant pump laser was $\mathrm{OFF}$ or $\mathrm{ON}$ in the pump-probe sequence presented underneath the curve Fig. 2(b). The difference of the two PL signals reflects the population difference 
between a sequence with optical pumping and a sequence where $S_{z}=-5 / 2$ is evenly populated. The height of the difference signal at the beginning of the probe pulse, which reaches $75 \%$ gives a direct measurement of the efficiency of the spin optical pumping. The PL transients observed during the probe pulse corresponds to the progressive destruction of the nonequilibrium distribution prepared by the pump. This reset process is produced by the injection of unpolarized excitons and its rapidity depends on the intensity of the probe laser.

A more direct way to probe the optical pumping process is to monitor the time evolution of the fluorescence signal observed during the resonant excitation. Excitation transfer can occur within the X-Mn complex during the lifetime of the exciton and gives rise to a weak PL on all the QDs levels. Whatever the spin-relaxation processes involved in this excitation transfer, this signal depends on the absorption of the pump laser which is controlled by the occupation of $S_{z}=-5 / 2:$ it monitors the spin selective absorption of the QD and is then a direct probe of the pumping efficiency of the $\mathrm{Mn}$ spin. The pumping efficiency is then given by $\Delta I / I_{0}$ $\approx 75 \%$ (see Fig. 2), in agreement with the pumping efficiency measured on the probe sequence.

The time evolution of the PL detected on the low-energy state of X-Mn under a resonant excitation on the high-energy state is presented in Figs. 2(b) and 2(c) for two different pump-probe sequences: probe $\mathrm{ON}$ and probe OFF, respectively. When the probe laser is switched $\mathrm{ON}$ in the pumpprobe sequence, an equilibrium distribution of the Mn spin is restored by the nonresonantly injected unpolarized excitons before each pumping pulse. The absorption, and then the amplitude of the resonant fluorescence signal is maximum at the beginning of the pump pulse and progressively decreases as the state $S_{z}=-5 / 2$ is emptied by the optical pumping process. When the probe laser is switched OFF in the pumpprobe sequence, the resonant fluorescence transients during the pump pulse disappear and a weak constant PL is observed. This disappearance of the transient is a signature of the perfect conservation of the Mn spin distribution during the dark time between each pumping pulse. The steady state PL depends on the optical pumping efficiency which is controlled by the ratio of the relaxation rates for the Mn spin in the exchange field of the exciton and the relaxation and coherent evolution of the Mn spin in an empty dot. ${ }^{14,16}$

\section{TIME-RESOLVED RESONANT FLUORESCENCE}

The resonant fluorescence signal can be used to analyze the influence of the excitation intensity, wavelength, and polarization on the efficiency of the Mn spin optical pumping. A detail of the time-resolved resonant fluorescence signal obtained with the pump laser tuned strictly on resonance with the high-energy level is presented in Fig. 3(b). A decrease of about $75 \%$ of the resonant PL is observed during the optical pumping process with a characteristic time of $\tau_{\text {pump }}=70 \mathrm{~ns}$. This exponential decay reflects the decrease in the absorption of the QD induced by the decrease in the population of the state $S_{z}=-5 / 2$ and shows it takes a few tens of nanoseconds to initialize the Mn spin. Alternatively,
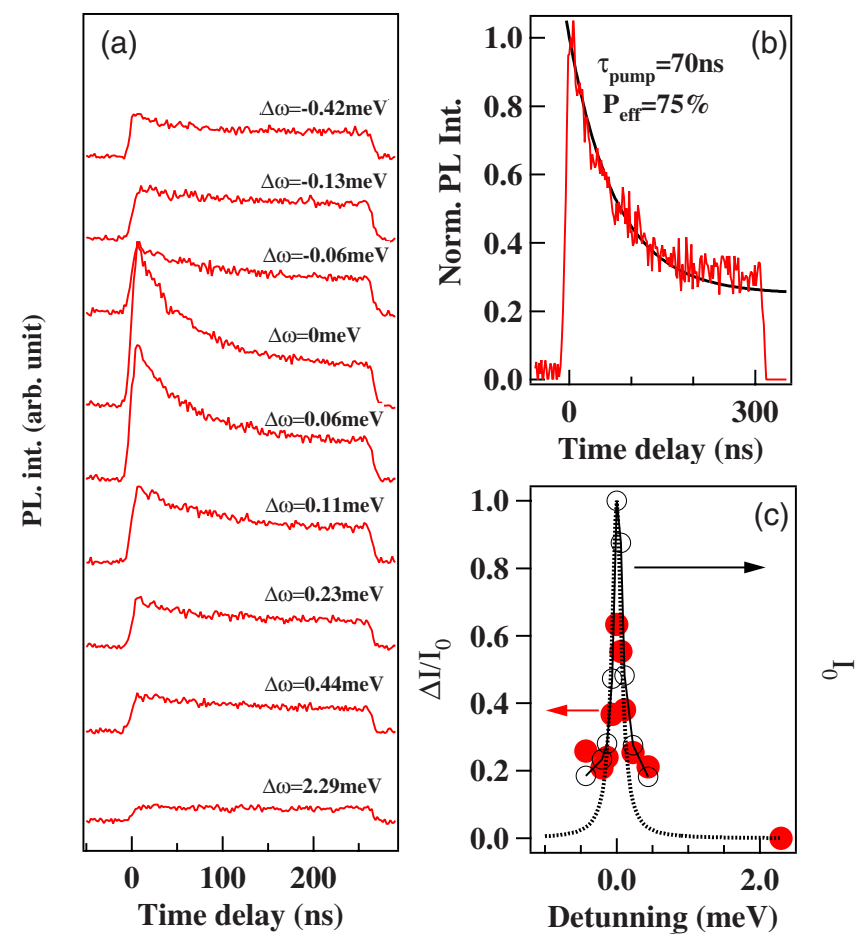

FIG. 3. (Color online) (a) Excitation energy dependence of the resonant fluorescence signal obtained on QD1 for cross-circular excitation detection on the high- and low-energy exciton lines, respectively (positive detuning corresponds to an excitation on the highenergy side of the line). (b) Detail of the resonant fluorescence transient recorded during the optical pumping process. This typical time-resolved resonant fluorescence spectrum is obtained with an integration time of $600 \mathrm{~s}$ and a count rate of about $500 \mathrm{cts} / \mathrm{s}$. The exponential fit (black line) gives an optical pumping efficiency $P_{\text {eff }} \approx 75 \%$ and a pump time of $70 \mathrm{~ns}$. (c) Amplitude of the resonant fluorescence signal as the excitation is tuned around the high energy line of X-Mn. The Lorentzian fit give a full width at half maximum of $80 \mu \mathrm{eV}$.

one can say that the transition can be recycled for a few tens of nanoseconds before the laser induces a Mn spin-flip event. After a few tens of nanoseconds the PL reaches a steadystate intensity. This resonant fluorescence signal can be used to analyze in detail the optical pumping mechanism.

Figures 3(a) and 3(b) present the amplitude and time evolution of the fluorescence signal detected on $\left|+1, S_{z}=-5 / 2\right\rangle$ for different pump wavelength around the high-energy level $\left|-1, S_{z}=-5 / 2\right\rangle$. A clear resonant behavior is observed in the initial amplitude $I_{0}$ of the fluorescence signal [Fig. 3(c)]. This reflects the wavelength and excitation power dependence of the absorption of the QD. The measured width of the resonance $(\sim 80 \mu \mathrm{eV})$ is a convolution of the width of the QD's absorption in the nonlinear regime and of the linewidth of the excitation laser $(\sim 60 \mu \mathrm{eV})$. The efficiency of the optical pumping $\Delta I / I_{0}$, presents a similar resonance demonstrating the strong excitation energy dependence of the spin optical pumping process.

Figure 4(a) presents the time-resolved PL recorded on the low-energy line during the pump and the probe pulses detected in the two circular polarizations. For cross circularly polarized pump excitation and PL detection, the PL probes 

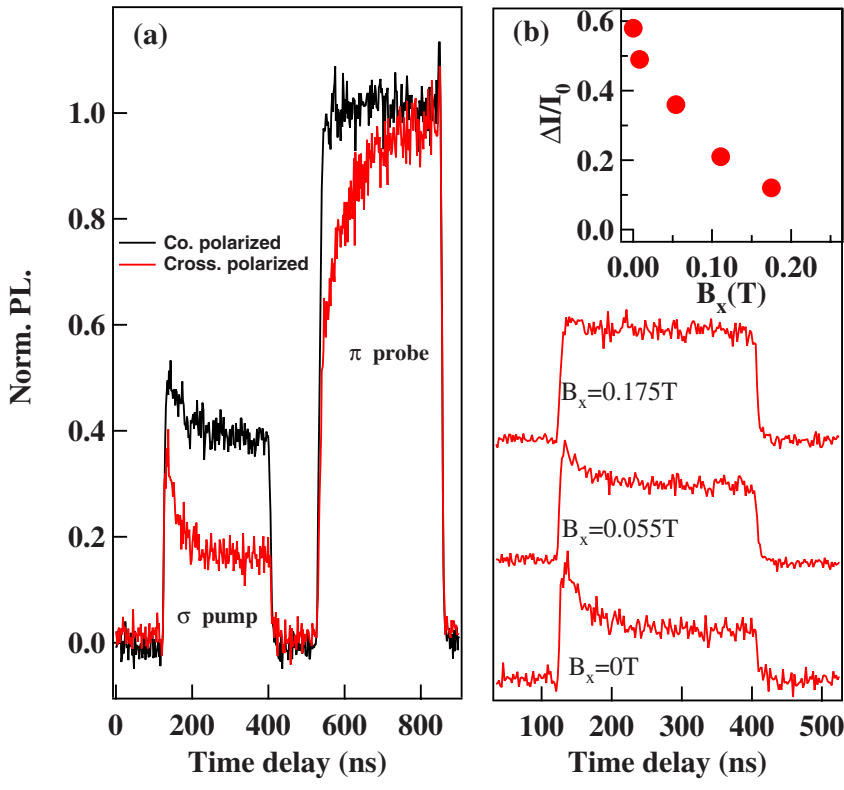

FIG. 4. (Color online) (a) Circularly polarized PL obtained on QD1 during the optical pumping sequence for cocircularly and cross-circularly polarized pump. (b) Transverse magnetic field $\left(B_{x}\right)$ dependence of the resonant fluorescence signal under crosspolarized excitation detection. The inset presents the amplitude of the optical pumping signal as a function of transverse magnetic field.

the Mn spin state resonantly excited by the pump laser $\left(S_{z}\right.$ $=-5 / 2$ for $\sigma$ pump). In this case, the pumping effect is both observed in the transient of the resonant fluorescence and as a decrease in the initial PL intensity in the probe signal. For copolarized pump excitation and PL detection $(\sigma-)$, the PL intensity of the low-energy line is proportional to the population of $S_{z}=+5 / 2$. As evidenced by the PL of the probe pulse, the population of this state is not significantly affected by the resonant pump laser. The resonant PL during the pump is dominated by the direct absorption in the lowenergy line acoustic phonon side band ${ }^{19}$ and the transient caused by the depletion of $S_{z}=-5 / 2$ can be hardly observed. As expected for a Mn pumping process, the influence of a $\sigma$-resonant laser tuned on the high-energy X-Mn level mainly affects the population of the state $S_{z}=-5 / 2$.

More information on the pumping process can be obtained from the transverse magnetic field dependence of the time-resolved resonant fluorescence signal. The precession of the Mn spin in a transverse magnetic field decreases the probability of conserving a prepared $S_{z}$ state. For an isotropic $\mathrm{Mn}$ spin, the damping of the precession induced by the decoherence should give rise to the standard Hanle depolarization curve with a Lorentzian shape and a width proportional to $1 / T_{2},{ }^{20}$ where $T_{2}$ is the decoherence time of Mn spin. In the present case, an in-plane magnetic field $B_{x}$ induces coherent precession of the $\mathrm{Mn}$ spin away from the optical axis (=QDs' growth axis) so that the average spin polarizatizon, and therefore the amplitude of the optical pumping signal, decays [Fig. 4(b)]. However, a strain-induced magnetic anisotropy along the growth axis ${ }^{14,21}$ partially blocks the $\mathrm{Mn}$ spin precession. As already observed in the optical orienta-
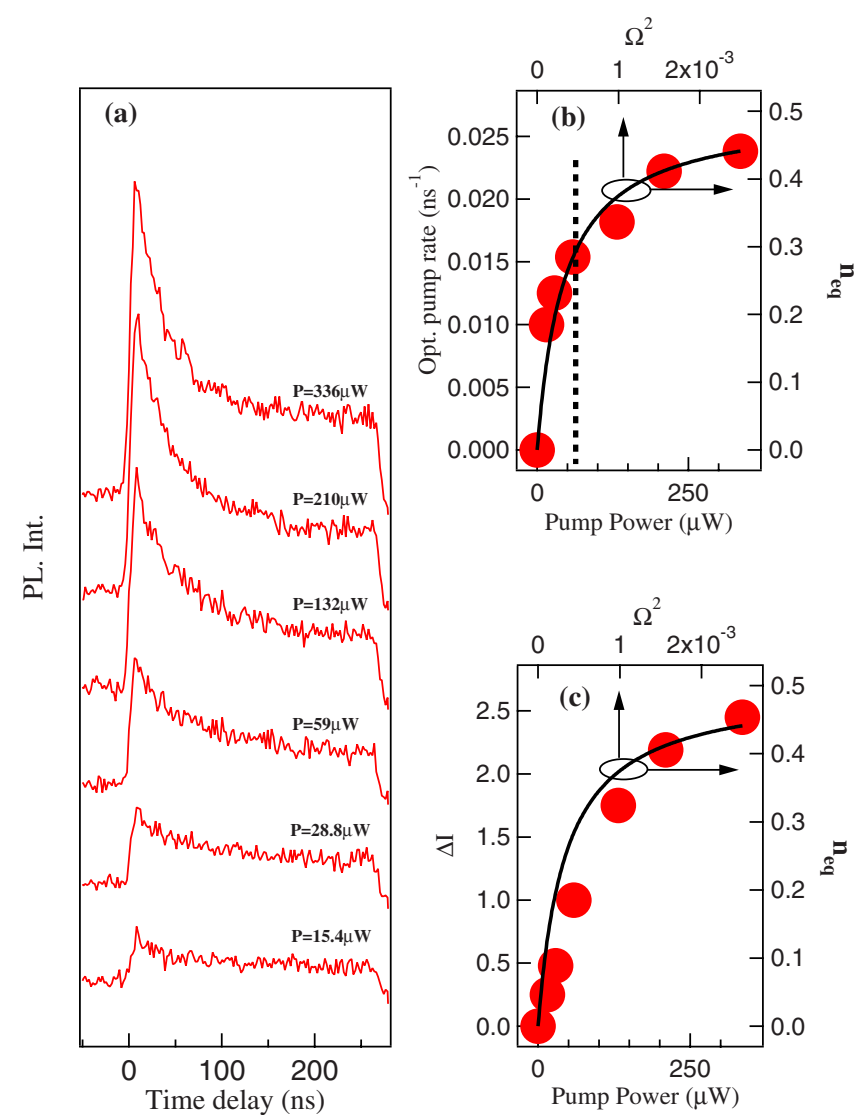

FIG. 5. (Color online) (a) Excitation power dependence of the resonant fluorescence signal of QD1. (b) Excitation power dependence of the optical pumping rate. The vertical dotted line shows the excitation power range used in the optical pumping wavelength dependence presented in Fig. 3. (c) Excitation power dependence of the amplitude of the optical pumping signal. The solid lines in (b) and (c) represent the calculated evolution of the population of a resonantly excited two level system as a function of the square of the Rabi frequency which is proportional to excitation intensity. In this calculation, $T_{1}=180 \mathrm{ps}, T_{2}=10 \mathrm{ps}$, and $\Omega^{2}$ is scaled to reproduce the observed saturation.

tion experiments under nonresonant excitation (see Ref. 19), the transverse magnetic field decreases the efficiency of the optical pumping [inset of Fig. 4(b)]. This field dependence is not controlled by the Mn coherence time but mainly by its magnetic anisotropy and is a probe of the strain state of the QD at the Mn location.

As displayed in Fig. 5, the characteristic time and the amplitude of the optical pumping signal also depends on the excitation intensity. In the low excitation regime, as expected for a spin optical pumping process, the transient characteristic time $\left(\tau_{\text {pump }}\right)$ is inversely proportional to the pump laser intensity. However, a saturation behavior is clearly observed both for the amplitude and the characteristic time of the resonant fluorescence transient. Here, the rate of the spin optical pumping saturates around $0.025 \mathrm{~ns}^{-1}$ [Fig. 5(b)]. The saturation of the optical pumping process results from a saturation of the absorption of the resonantly excited excitonic level. Indeed, the population of a two level system driven by a resonant excitation laser is given by ${ }^{22}$ 


$$
n_{e q}=\frac{1}{2} \frac{\Omega^{2}\left(\frac{T_{1}}{T_{2}}\right)}{\left(\Delta \omega^{2}+\frac{1}{T_{2}^{2}}+\Omega^{2} \frac{T_{1}}{T_{2}}\right)},
$$

where $\Omega$ is the Rabi frequency, $\Delta \omega$ the detuning between the excitation laser and the excitonic transition, and $T_{1}$ and $T_{2}$ the lifetime and the coherence time of the exciton, respectively. The rate of the spin optical pumping process, which is proportional to $n_{e q}$, is expected to increase with the excitation Rabi frequency until it reaches a saturation value when the Rabi frequency is larger than the spontaneous emission rate $\left(\Omega \gg T_{1}^{-1}\right)$. The saturation curve obtained with Eq. (1), $T_{1}=180 \mathrm{ps}, T_{2}=10 \mathrm{ps},{ }^{23}$ and $\Delta \omega=0$ is compared with the optical pumping signal in Figs. 5(b) and 5(c). A good agreement with this simple model describing the population of a two level system resonantly excited by a cw laser is obtained.

In the saturation regime, if $S_{z}=-5 / 2$ the QD is in the $\left|-1, S_{z}=-5 / 2\right\rangle$ state half of the time in average. Taking for granted that $\Gamma_{\mathrm{Mn}, \mathrm{X}} \gg \Gamma_{\mathrm{Mn}, G}$, the rapidity of the optical pumping process is no longer controlled by the rate at which excitons are injected but depends only on the relaxation rate from the state $\left|-1, S_{z}=-5 / 2\right\rangle$ to other X-Mn levels with $S_{z}$ $\neq-5 / 2$. Therefore, the pumping rate in the saturation regime gives an estimation of the spin-flip rate of the $\mathrm{Mn}$ in the exchange field of the exciton $\Gamma_{\mathrm{Mn}, \mathrm{X}} / 2 \approx \Gamma_{\text {pump }}$ and a relaxation time $\tau_{\mathrm{Mn}, \mathrm{X}} \approx 20 \mathrm{~ns}$ in agreement with the value deduced from photon correlation measurements. ${ }^{7}$

\section{DYNAMICS OF EXCITON AND Mn SPINS}

Information about the spin-relaxation mechanism of the exciton exchange coupled with a $\mathrm{Mn}$ spin can be obtained from the energy of the resonant fluorescence signal. The spectral distribution of the scattered photons observed during the resonant excitation on the QD ground state results from spin-flip processes within the exciton-Mn systems. A simple thermalization among the 24 exciton-Mn levels (see Ref. 12) should give rise to a thermal distribution of the intensities of the PL lines in the resonant fluorescence spectrum.

Here, we report results on a QD presenting a weak optical pumping at $B=0 \mathrm{~T}$. This poor efficiency of optical pumping could be attributed to the local strain environment which leads to $\Gamma_{\mathrm{Mn}, \mathrm{X}} \approx \Gamma_{\mathrm{Mn}, G}$. This case is interesting for a study of the X-Mn dynamics: the QD line under excitation is always absorbant and the scattered photons reflect the fastest spinrelaxation channels. Figure 6(a) presents the three photoluminescence excitation spectra (PLE) detected on the lowenergy lines of X-Mn which are associated with the Mn spin states $+5 / 2,+3 / 2$, and $+1 / 2$ (plain lines). In addition, PLE spectra detected on a dark exciton state associated with the Mn spin $\pm 1 / 2$ is also presented (dotted line). The corresponding resonant PL spectra obtained at the maximum of the PLE signal are also displayed. Finally, a PL spectrum in quasiresonant excitation is displayed at the bottom of Fig. 6(a). The QD's spectrum differs from the one expected in the heavy-hole approximation and presents seven lines. Such PL
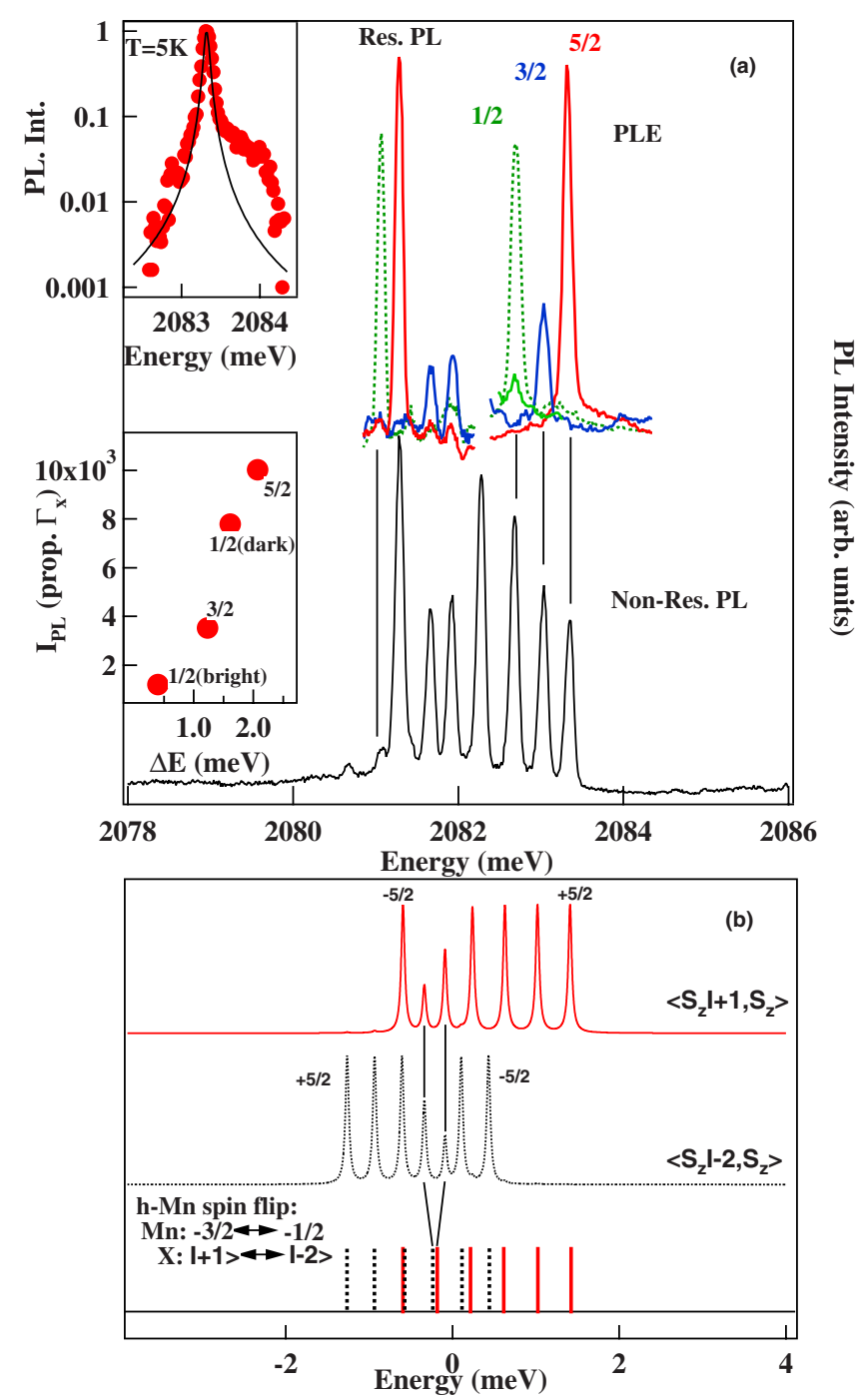

FIG. 6. (Color online) PLE spectra and resonant photoluminescence obtained on the ground state of a Mn-doped QD (QD2). The resonant PL is obtained under circularly cross-polarized excitation detection. The spin state of the Mn is conserved during the spin flip of the exciton. The top inset presents an enlarge view of the PLE obtained under excitation and detection on the $S_{z}=+5 / 2$ state. The black line is a Lorentzian fit with a half width at half maximum of $80 \mu \mathrm{eV}$. The asymmetry of the absorption line comes from a coupling with acoustic phonons. The bottom inset displays the intensity of the resonant PL $\left(I_{\mathrm{PL}}\right)$ obtained for excitation on the different Mn spin states as a function of the energy splitting of the corresponding exciton-Mn levels. (b) Calculated bright $|+1\rangle$ and dark $|-2\rangle$ energy levels with $J_{e \mathrm{Mn}}=-0.06 \mathrm{meV}, \quad J_{h \mathrm{Mn}}=0.245 \mathrm{meV}$, $J_{e h}=-0,550 \mathrm{meV}$, and $\epsilon_{v b m}=0.1$. Because of the large carrier-Mn exchange coupling, the dark exciton levels overlap with the bright excitons. The valence-band mixing coupled the states $|+1,-3 / 2\rangle$ and $|-2,-1 / 2\rangle$, and gives rise to an additional PL line. The bottom scheme presents the energy of the uncoupled X-Mn levels.

spectrum appears when the high-energy lines of the dark exciton states overlap the low-energy lines of the bright exciton states. Strain induced VBM allows simultaneous hole-Mn spin flips and couples dark and bright states. If the states coupled through these spin flips are close in energy in the heavy-hole approximation, this leads to a strong mixing 
of a bright state with a dark state and the QD presents additional PL lines. This is the case for the QD presented in Fig. 6(a): VBM mixes $|+1,-3 / 2\rangle$ with $|-2,-1 / 2\rangle$ and the new eigenstates share the oscillator strength of the bright state $|+1,-3 / 2\rangle$. The two lines on the right of the low-energy state can be attributed to the bright part of the states $\alpha \mid+1$, $-3 / 2\rangle+\beta|-2,-1 / 2\rangle$ and $\beta|+1,-3 / 2\rangle+\alpha|-2,-1 / 2\rangle$, with $|\alpha|$ and $|\beta|$ close to $1 / 2$. The two small peaks at low energy come from dark states with a weaker bright exciton component. The attribution of these lines to the bright and dark exciton states is confirmed by the calculation of the energy levels presented in Fig. 6(b) following Refs. 6 and 24.

From these PLE spectra and resonant PL spectra, it follows that the most efficient spin-relaxation channels within the X-Mn system do conserve the Mn spin. For instance, an excitation with $\sigma+$ photons on the high-energy line (state $\left.\left|+1, S_{z}=+5 / 2\right\rangle\right)$ produces $\sigma-$ PL mainly on the low-energy state $\left|-1, S_{z}=+5 / 2\right\rangle$. A similar behavior is observed for an excitation on $\left|+1, S_{z}=+3 / 2\right\rangle$ which generate a PL on $\mid-1, S_{z}$ $=+3 / 2\rangle$. In both cases, this excitation transfer corresponds to a spin flip of the exciton from $|+1\rangle$ to $|-1\rangle$ with a conservation of the Mn spin.

More surprising, an excitation on $\left|+1, S_{z}=+1 / 2\right\rangle$ produces the strongest PL on an exciton level formed mostly of a dark state with a weak oscillator strength [weak signal in the nonresonant PL plotted at the bottom of Fig. 6(a)] with the same Mn spin $\left(S_{z}=+1 / 2\right)$. This confirms the good stability of the $\mathrm{Mn}$ spin under these resonant excitation conditions and the efficient spin flip of the exciton in the exchange field of the Mn.

In the spin-flip scattering processes which conserve the Mn spin, the hh exciton stays in the same spatial state and just flips its spin (i.e., the electron and the hole spin flip simultaneously), by simultaneously emitting (or, at high temperature, absorbing) an acoustic phonon. This can occur via the $\mathrm{lh}$ and hh exciton mixing due to the interplay of the short-range exchange interaction and the lattice deformation. ${ }^{6}$ Such single phonon process is responsible for an increase in the exciton spin-relaxation rate observed in small QDs with the increase in the Zeeman splitting $\Delta E .^{24-26}$

For typical QDs and low temperature, the zero magnetic field exciton spin-relaxation time is longer than the exciton radiative lifetime and no exciton spin flip is expected during its lifetime. However for QDs with large short-range exchange interaction and small hh-lh splitting such as CdTe/ ZnTe QDs, ${ }^{6}$ an increase in the exciton splitting can significantly shorten this relaxation time. ${ }^{24,26}$ This faster spinrelaxation rate is a consequence of the increase in the acoustic phonon density of states at the energy of the interlevel splitting.

In Mn-doped QDs, the exciton Mn exchange interaction acts on the exciton as an effective magnetic field, aligned along the QDs' growth axis, which increases with $S_{z}$. With values of $\Delta_{s t} \sim 1 \mathrm{meV}, \Delta_{\mathrm{lh} \text { hh }} \sim 30 \mathrm{meV}$ (Ref. 6) and an interlevel splitting $\Delta E=2 \mathrm{meV}$ for the $S_{z}= \pm 5 / 2$ states in QD2, we estimate according to Ref. 17, an exciton spin lifetime of about $0.4 \mathrm{~ns}$. The large exciton splitting leads to an exciton spin lifetime that can reach the exciton lifetime scale $\approx 0.2 \mathrm{~ns}$. This decrease in the exciton spin lifetime, which scales as $(\Delta E)^{-3}$ (according to Ref. 22), can explain the ob-

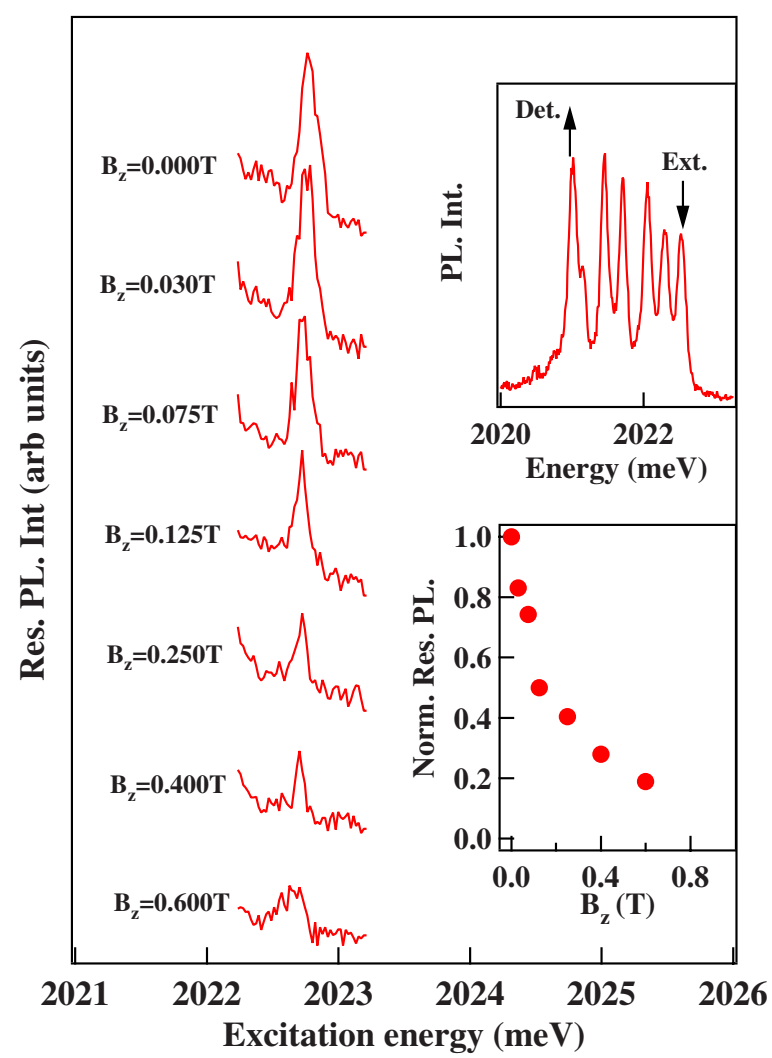

FIG. 7. (Color online) Magnetic field dependence of the PLE spectra obtained on the ground state of a Mn-doped QD (QD3) under circularly cross-polarized excitation detection. The insets present a PL spectra of QD3 (arrows point the excitation and detection wavelength) and the magnetic field $\left(B_{z}\right)$ dependence of the amplitude of the resonant PL signal.

served efficient transfer of excitation within the X-Mn complex. This splitting dependence of the exciton relaxation rates can also explain the decrease in the amplitude of the PLE signal with the decrease of $S_{z}$ [inset of Fig. 6(a)]: a decrease in the splitting reduces the spin-flip rate and consequently the efficiency of the exciton transfer. Similarly, when exciting the state $\left|+1, S_{z}=+1 / 2\right\rangle$, the exciton preferentially relaxes to the dark exciton level $\left|-2, S_{z}=+1 / 2\right\rangle$ through a spin flip of the hole, at lower energy than the bright exciton $\left|-1, S_{z}=+1 / 2\right\rangle$. Despite the weak oscillator strength of this level, most of the resonant PL is then observed on this lowenergy state.

For a given Mn spin state, the amplitude of the resonant fluorescence depends on an applied external magnetic field. This is illustrated in Fig. 7 where the PLE spectra detected on the low-energy line when the excitation laser is tuned around the high-energy level are presented for different magnetic fields in Faraday geometry. The PL intensity is divided by two for a magnetic field of about $100 \mathrm{mT}$. This reduction could either be explained by a reduction in the spin-flip rate of the exciton or an increase in the efficiency of the optical pumping of the Mn spin. The Zeeman energy of the exciton in this weak magnetic field is not significant compared to the exchange field with Mn: the dynamics of the exciton coupled with the $\mathrm{Mn}$ is unlikely to be affected by this small energy change. Thus, the magnetic field dependence is attributed to 

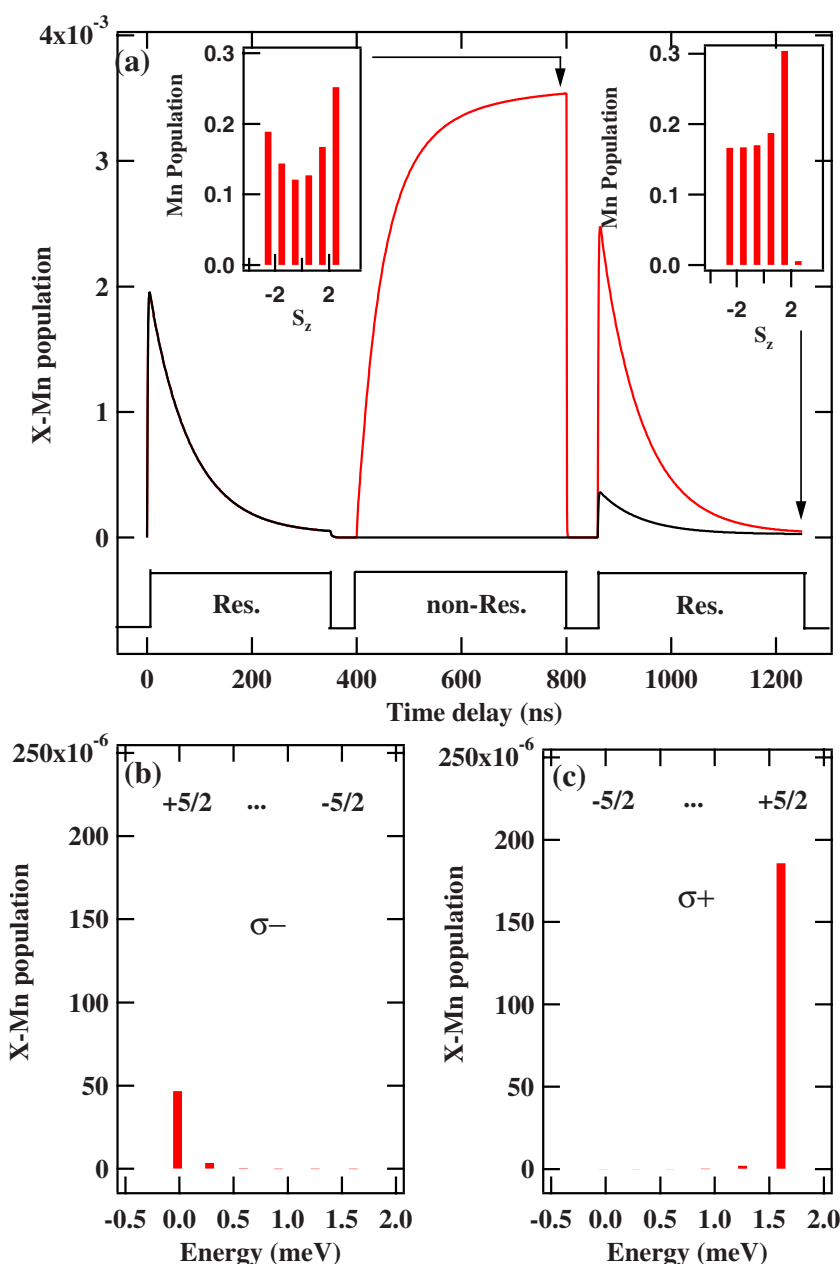

FIG. 8. (Color online) (a) Calculated time-resolved PL of the low-energy line of a Mn-doped QD under the excitation sequence used in the resonant pumping experiments (see Fig. 2). [(b) and (c)] Steady state population of the $|-1\rangle$ and $|+1\rangle$ excitons, respectively, at the end of the resonant pumping pulse on $\left|+1, S_{z}=+5 / 2\right\rangle$. The level are displayed as a function of there energy. The parameters used in the calculation are: $J_{e \mathrm{Mn}}=-0.05 \mathrm{meV}, J_{h \mathrm{Mn}}=0.2 \mathrm{meV}$, $J_{e h}=-0.5 \mathrm{meV}, \quad D_{0}=0.007 \mathrm{meV}, \quad T=6 \mathrm{~K}, \quad \Gamma_{e}=0.1 \mathrm{~ns}^{-1}, \quad \Gamma_{h}$ $=0.1 \mathrm{~ns}^{-1}, \Gamma_{\mathrm{Mn}, \mathrm{X}}=0.05 \mathrm{~ns}^{-1}, \Gamma_{\mathrm{Mn}, G}=0.0001 \mathrm{~ns}^{-1}, \Gamma_{b}=4 \mathrm{~ns}^{-1}, \Gamma_{d}$ $=0.1 \mathrm{~ns}^{-1}, \Gamma_{\text {Pump }}=0.5 \mathrm{~ns}^{-1}$, and $\Gamma_{\text {Probe }}=0.05 \mathrm{~ns}^{-1}$.

an increase in the optical pumping efficiency. Indeed, the Zeeman splitting of the Mn in an empty dot cancels the nondiagonal coupling induced by the tetragonal crystal field or an anisotropic strained distribution at the $\mathrm{Mn}$ atom location. ${ }^{14,21}$ It improves the $\mathrm{Mn}$ spin conservation thus accounting for the increase in the optical pumping efficiency in a weak magnetic field.

\section{Mn SPIN DYNAMICS AND OPTICAL PUMPING MECHANISM}

In the absence of carriers, the dominant spin-relaxation mechanism for the $\mathrm{Mn}$ is the coupling to acoustic phonons. The relaxation rate is then proportional to the density of states of phonons at the energy of the spin transition. Thus, the larger the energy difference between initial and final spin states, the larger the spin-flip rate. ${ }^{25}$ For instance, for diluted $\mathrm{Mn}$ spins under large magnetic field, a $B^{5}$ increase in the spin-relaxation rate has been reported. ${ }^{27}$

At zero magnetic field, Mn spin transitions in the ground state have an energy splitting on the order of the strained induced magnetic anisotropy $D_{0} \approx 7 \mu \mathrm{eV} .{ }^{14}$ In the presence of the exciton, however, the Mn splitting is of the order of $J_{h \mathrm{Mn}}$ which is at least ten times larger. This should give relaxation rates five orders of magnitude larger. In QDs with in-plane biaxial strains, the inequality $\Gamma_{\mathrm{Mn}, G} \ll \Gamma_{\mathrm{Mn}, \mathrm{X}}$ is then easily verified and the mechanism of Mn-spin pumping is relatively insensitive to the value of the $\mathrm{Mn}$ spin lifetime $\Gamma_{\mathrm{Mn}, G}$

To model the optical pumping process, we propose a master equation for the occupation of the eigenstates of a simplified single Mn-doped Hamiltonian QD that features the projection along the $z$ axis of the spin-1/2 electron $S^{e}$, the pseudospin $J=3 / 2, J_{z}= \pm 3 / 2$ of the heavy hole and the spin $5 / 2$ of the $\mathrm{Mn}$

$$
\mathcal{H}=J_{h \mathrm{Mn}} S_{z}^{h} S_{z}^{\mathrm{Mn}}+J_{e h} S_{z}^{h} S_{z}^{e}+J_{e \mathrm{Mn}} S_{z}^{e} S_{z}^{\mathrm{Mn}}+D_{0}\left(S_{z}^{\mathrm{Mn}}\right)^{2} .
$$

The first term corresponds to the hole-Mn coupling, which is antiferromagnetic $\left(J_{h \mathrm{Mn}}>0\right)$. The second to the electron-hole exchange, which is ferromagnetic, so that the dark \pm 2 excitons lie below the bright \pm 1 exciton doublet. The third term is the electron-Mn exchange. The fourth term is the single ion anisotropy adequate for a strained thin layer. ${ }^{21}$ The spinflip contributions, present in the $e$-Mn case and, depending on the dot shape, in the $e-h$ and $h$-Mn coupling, have been studied in detail elsewhere ${ }^{28}$ and, in first approximation, can be neglected to model the optical pumping process at zero magnetic field. Hamiltonian (2) commutes with $S_{z}^{\mathrm{Mn}}, S_{z}^{e}$, and $S_{z}^{h} \quad$ so that the eigenvalues are $E\left(S_{z}^{\mathrm{Mn}}, S_{z}^{e}, S_{z}^{h}\right)=E_{\mathrm{X}}$ $+J_{h \mathrm{Mn}} S_{z}^{h} S_{z}^{\mathrm{Mn}}+J_{e h} S_{z}^{h} S_{z}^{e}+J_{e \mathrm{Mn}} S_{z}^{e} S_{z}^{\mathrm{Mn}}$, where $E_{\mathrm{X}} \simeq 2 \mathrm{eV}$ is the bare exciton energy. To reproduce typical experimental spectrum we take $J_{e \mathrm{Mn}}=0.05 \mathrm{meV}, J_{h \mathrm{Mn}}=0.2 \mathrm{meV}$, and $J_{e h}=-0.5 \mathrm{meV}^{3}{ }^{3}$ The corresponding spectrum is made of six doublets for the bright exciton above the six doublets for the dark excitons. The ground-state spectrum is given by $D_{0}\left(S_{z}^{\mathrm{Mn}}\right)^{2}$ with $D_{0}=7 \mu \mathrm{eV} .{ }^{21}$

The master equation model that we use here is an extension of the model by Govorov and Kalameitsev (hereafter GK) (Ref. 15) in their proposal of optical spin pumping of a single $\mathrm{Mn}$ in a QD. In GK, a unique rate is assigned to transitions between the 24 exciton levels, complying with principle of detailed balance but neglecting the dependence of the rates on energy and spin change. Here we propose a model in which transitions are only permitted between states that are connected via the flip of a single spin (the one of either the Mn, the electron or the hole). This rule is certainly valid in most of spin-relaxation mechanisms and restricts significantly the relaxation pathways. We still neglect the dependence of the rates on the energy difference, except for the fact that we use the principle of detailed balance. Thus, our model has four elementary rates $\Gamma_{e}, \Gamma_{h}, \Gamma_{\mathrm{Mn}, \mathrm{X}}$, and $\Gamma_{\mathrm{Mn}, G}$ for the relaxation of the spin of the electron, hole, $\mathrm{Mn}$ in the presence of the exciton and $\mathrm{Mn}$ alone. In addition, $S_{z}^{\mathrm{Mn}}$ conserving transitions between the six ground states and the 12 bright excitons transitions are described with a laser pump- 
ing function $g\left(S_{z}^{\mathrm{Mn}}, X\right)$ where $X= \pm 1$ labels the bright excitons. We consider that dark excitons are not created under these excitation conditions and take $g\left(S_{z}^{\mathrm{Mn}}, X= \pm 2\right)=0$. Mn spin conserving spontaneous emission from bright $\Gamma_{b}$ and dark $\Gamma_{d}$ are also included.

In the weak excitation regime, the PL and optical pumping process are described by the master equation

$$
\frac{d P_{n}}{d t}=\sum_{n^{\prime}}\left(\Gamma_{n^{\prime} \rightarrow n} P_{n^{\prime}}\right)-\left(\sum_{n^{\prime}} \Gamma_{n \rightarrow n^{\prime}}\right) P_{n}
$$

$n$ and $n^{\prime}$ denoting the 24 exciton-Mn eigenstates and the six Mn spin states. We consider that these spin flips are triggered by the emission or absorption of single acoustic phonons and are then thermally activated. Here we use $\Gamma_{i, n \rightarrow n^{\prime}}=\Gamma_{i}$ if $E_{n, n^{\prime}}=E_{n^{\prime}}-E_{n} \leq 0$ and $\Gamma_{i, n \rightarrow n^{\prime}}=\Gamma_{i} e^{-E_{n n^{\prime}} k_{B} T}$ if $E_{n, n^{\prime}}>0$ with $i=e, h, \mathrm{Mn}(\mathrm{X})$, or $\mathrm{Mn}$ and $E_{n}$ the eigenenergies of the Hamiltonian (2).

The model permits to consider the two excitation modes used in the pump-probe experiment: (i) resonant, for which $g\left(S_{z}^{\mathrm{Mn}}, X\right)$ is nonzero for only one of the 12 bright excitons states and (ii) unpolarized and quasiresonant for which $g\left(S_{z}^{\mathrm{Mn}}, X\right)$ is nonzero for all the 12 bright states. The timeresolved fluorescence signal detected on the low-energy line of X-Mn calculated using the above rate equation model is presented in Fig. 8(a). The excitation conditions used in the calculation presented in Fig. 8(a) reproduce the pump-probe sequence of the time-resolved optical pumping experiments displayed in Fig. 2. Values of the parameters are listed in the caption of Fig. 8. The exchange integrals are chosen to reproduce typical exciton-Mn spectra and values for $\Gamma_{\mathrm{Mn}, \mathrm{X}}, \Gamma_{b}$, and $\Gamma_{d}$ where deduced from time-resolved PL and autocorrelation measurements. ${ }^{7}$ A ratio $\Gamma_{\mathrm{Mn}, X} / \Gamma_{\mathrm{Mn}, G} \approx 500$ is enough to explain the observed optical pumping efficiency. The characteristic time of the optical pumping is also well reproduced with a generation rate $\Gamma_{\text {Pump }}$ which drives the QD close to the saturation.
Figures 8(b) and 8(c) present the steady-state population of the $|-1\rangle$ and $|+1\rangle$ excitons respectively at the end of a resonant pumping pulse on $\left|+1, S_{z}=+5 / 2\right\rangle$. This population is proportional to the PL intensity of the bright exciton levels. The introduction in the model of independent spin-flip rates for the confined carriers and the Mn qualitatively explain the observed resonant fluorescence spectra: the scattering spinflip processes mainly conserve the Mn spin and an excitation on $\left|+1, S_{z}^{\mathrm{Mn}}=+5 / 2\right\rangle$ empty the state $S_{z}^{\mathrm{Mn}}=+5 / 2$ and induces preferentially PL on $\left|+1, S_{z}^{\mathrm{Mn}}=+5 / 2\right\rangle$ and $\left|-1, S_{z}^{\mathrm{Mn}}=+5 / 2\right\rangle$.

\section{CONCLUSION}

In summary, our results demonstrate the spin optical pumping of a single magnetic atom in a semiconductor host. Resonant excitation of a Mn-doped QD ground state permits a high fidelity preparation of the Mn spin. We show that spin-flip-scattered photons can be used to probe the dynamics of the initialization of the Mn spin during the optical pumping process. The technique introduced here allows for an accurate and direct observation of the exciton spin-flip scattering. These measurements confirms that, as far as the spin dynamics is concerned, the Mn acts on the exciton as an effective magnetic field which increase the probability of single-phonon-induced spin flips. A more efficient Mn spin readout could be obtained monitoring directly the photons resonantly scattered by the optically driven excitonic level.

\section{ACKNOWLEDGMENTS}

The authors thank J. Cibert and D. Ferrand for fruitful discussions. This work is supported by the French ANR contract QuAMOS and Fondation Nanoscience (RTRAGrenoble). J.F.R. acknowledges funding from MEC-Spain (Grants No. MAT07-67845 and CONSOLIDER No. CSD2007-0010). *lucien.besombes@grenoble.cnrs.fr

${ }^{1}$ D. Press, T. D. Ladd, B. Zhang, and Y. Yamamoto, Nature (London) 456, 218 (2008).

${ }^{2}$ C. Latta, A. Högele, Y. Zhao, A. N. Vamivakas, P. Maletinsky, M. Kroner, J. Dreiser, I. Carusotto, A. Badolato, D. Schuh, W. Wegscheider, M. Atature, and A. Imamoglu, Nat. Phys. 5, 758 (2009) and references therein.

${ }^{3}$ L. Besombes, Y. Léger, L. Maingault, D. Ferrand, H. Mariette, and J. Cibert, Phys. Rev. Lett. 93, 207403 (2004).

${ }^{4}$ A. Kudelski, A. Lemaitre, A. Miard, P. Voisin, T. C. M. Graham, R. J. Warburton, and O. Krebs, Phys. Rev. Lett. 99, 247209 (2007).

${ }^{5}$ D. E. Reiter, T. Kuhn, and V. M. Axt, Phys. Rev. Lett. 102, 177403 (2009).

${ }^{6}$ Y. Léger, L. Besombes, L. Maingault, and H. Mariette, Phys. Rev. B 76, 045331 (2007).

${ }^{7}$ L. Besombes, Y. Léger, J. Bernos, H. Boukari, H. Mariette, J. P. Poizat, T. Clement, J. Fernandez-Rossier, and R. Aguado, Phys.
Rev. B 78, 125324 (2008).

${ }^{8}$ F. Tinjod, B. Gilles, S. Moehl, K. Kheng, and H. Mariette, Appl. Phys. Lett. 82, 4340 (2003).

${ }^{9}$ L. Maingault, L. Besombes, Y. Léger, C. Bougerol, and H. Mariette, Appl. Phys. Lett. 89, 193109 (2006).

${ }^{10}$ A. V. Koudinov, I. A. Akimov, Yu. G. Kusrayev, and F. Henneberger, Phys. Rev. B 70, 241305(R) (2004).

${ }^{11}$ Solid immersion lens (SILs) are made of $\mathrm{KTaO}_{3}$ which is an isotropic cubic crystal with an index of 2.25 at $600 \mathrm{~nm}$ and no birefringence in the visible range.

${ }^{12}$ K. Koyama, M. Yoshita, M. Baba, and T. Suemoto, Appl. Phys. Lett. 75, 1667 (1999).

${ }^{13}$ V. Zwiller and G. Björk, J. Appl. Phys. 92, 660 (2002).

${ }^{14}$ C. Le Gall, L. Besombes, H. Boukari, R. Kolodka, J. Cibert, and H. Mariette, Phys. Rev. Lett. 102, 127402 (2009).

${ }^{15}$ A. O. Govorov and A. V. Kalameitsev, Phys. Rev. B 71, 035338 (2005).

${ }^{16}$ M. Goryca, D. Ferrand, P. Kossacki, M. Nawrocki, W. Pacuski, 
W. Maslana, J. A. Gaj, S. Tatarenko, J. Cibert, T. Wojtowicz, and G. Karczewski, Phys. Rev. Lett. 102, 046408 (2009).

${ }^{17}$ J. Dreiser, M. Atatüre, C. Galland, T. Müller, A. Badolato, and A. Imamoglu, Phys. Rev. B 77, 075317 (2008).

${ }^{18}$ B. D. Gerardot, D. Brunner, P. A. Dalgarno, P. Ohberg, S. Seidl, M. Kroner, K. Karrai, N. G. Stoltz, P. M. Petroff, and R. Warburton, Nature (London) 451, 441 (2008).

${ }^{19}$ L. Besombes, K. Kheng, L. Marsal, and H. Mariette, Phys. Rev. B 63, 155307 (2001).

${ }^{20}$ R. C. Myers, M. H. Mikkelsen, J.-M. Tang, A. C. Gossard, M. E. Flatté, and D. D. Awschalom, Nature Mater. 7, 203 (2008).

${ }^{21}$ M. Qazzaz, G. Yang, S. H. Xin, L. Montes, H. Luo, and J. K. Furdyna, Solid State Commun. 96, 405 (1995).
${ }^{22}$ E. B. Flagg, A. Muller, J. W. Robertson, S. Founta, D. G. Deppe, M. Xiao, W. Ma, G. J. Salamo, and C. K. Shih, Nat. Phys. 5, 203 (2009).

${ }^{23}$ B. Patton, W. Langbein, U. Woggon, L. Maingault, and H. Mariette, Phys. Rev. B 73, 235354 (2006).

${ }^{24}$ E. Tsitsishvili, R. V. Baltz, and H. Kalt, Phys. Rev. B 67, 205330 (2003).

${ }^{25}$ A. V. Khaetskii and Y. V. Nazarov, Phys. Rev. B 64, 125316 (2001).

${ }^{26}$ L. M. Woods, T. L. Reinecke, and Y. Lyanda-Geller, Phys. Rev. B 66, 161318(R) (2002).

${ }^{27}$ T. Strutz et al., Phys. Rev. Lett. 68, 3912 (1992).

${ }^{28}$ J. Fernández-Rossier, Phys. Rev. B 73, 045301 (2006). 